Neurosurg Focus 18 (4):E9, 2005

\title{
History of acoustic neurinoma surgery
}

\author{
Theofilos G. Machinis, M.D., Kostas N. Fountas, M.D., Ph.D., \\ Vassilios Dimopoulos, M.D., AND Joe Sam Robinson, M.D.
}

Department of Neurosurgery, The Medical Center of Central Georgia, Mercer University School of Medicine, Macon, Georgia

\begin{abstract}
The purpose of this article is to provide insight into the development of surgery for acoustic neurinomas throughout the years. The significant contribution of surgical authorities such as Cushing, Dandy, and House are discussed. The advances in surgical techniques from the very first operations for acoustic tumors at the end of the 19th century until today are described, with special emphasis on the technological and diagnostic milestones that preceded each step of this development.
\end{abstract}

\section{KEY WORDS • acoustic neurinoma • vestibular schwannoma • middle cranial fossa • retrosigmoid approach • translabyrinthine approach • history of neurosurgery}

The tumors variously known as acoustic neurinomas, acoustic neuromas, and vestibular schwannomas constitute an important intracranial pathological entity, accounting for 6 to $8 \%$ of all intracranial neoplasms and 80 to $90 \%$ of tumors of the cerebellopontine angle. ${ }^{12}$ Because they arise from the superior vestibular and not the acoustic division of the eighth cranial nerve, and are composed of Schwann cells in the neurilemma, the more accurate term "vestibular schwannoma" has been proposed. ${ }^{11,19}$ Nevertheless, the terms acoustic neuroma or neurinoma, which were based on the previous belief that these tumors develop from the acoustic nerve itself, are well embedded in the literature, and we will use these terms for historical consistency. The distinct clinical picture associated with vestibular schwannomas was noted quite early by the medical community, but the complex anatomy of this region of the brain and the intimate relationship among its structural components demanded great caution and stepwise development of surgical approaches, which occurred parallel to the advances of intraoperative technology and imaging techniques.

The first presumptive case of acoustic neurinoma dates back to the second half of the 18 th century. As Cushing ${ }^{8}$ related, in 1777, Sandifort "documented a small body adherent to the right auditory nerve," which was found at autopsy in a patient with deafness. Several reports on tumors possibly related to the auditory nerve followed, but it was not until 1830 that Charles Bell gave an accurate description, characteristic of the British physician of the time, of what appears to be a definitive case of acoustic neurinoma. A few years later, Cruveilhier published an outstanding and highly detailed report on the progression of clinical symptoms and the postmortem findings in a 26-year-old woman. Before autopsy, he had considered the base of the skull as the probable location of the tumor. Almost half a century after Cruveilhier's unique clinical description, Oppenheim in Germany achieved the first correct localization of an unambiguous tumor of the nervus acusticus based on its clinical symptoms.

During the last decades of the 19th century, advances in the histological and pathological characterization of tumors led to a more solid correlation of a patient's clinical symptoms with the actual diagnosis obtained at autopsy. Sternberg (1900) is credited with the first accurate pathological description of an acoustic neurinoma. Meanwhile, clinical observations by the leading physicians of that time, such as Babinski and Jackson, enabled a refinement of the knowledge of the functional anatomy of the brain, and in particular the cerebellar region, allowing a more accurate localization of pathological processes in that area based on the patient's symptomatology. Now that a presumptive diagnosis could be obtained during life, the time for intervention had arrived.

As Cushing wrote ${ }^{8}$, the first surgical procedure for a cerebellopontine angle tumor, a term introduced by Koch in the early 20th century, was most probably performed by von Bergmann in 1890; the patient died before the tumor could be localized. Five years later, Annandale in Edinburgh reported what appears to be the first successful surgical result in a patient with a tumor of the nervus acusticus. One year before, in 1894, Ballance ${ }^{1,24}$ in London had performed a successful two-stage operation for a tumor that he called an "encapsulated fibro-sarcoma." Careful review of his description of the tumor, however, raises the suspicion that it may have been an acoustic neurinoma (Cushing considered that very same tumor to be a case of meningeal endothelioma). Several reports of early treatment failure after surgery would follow, with failure most probably due to the preference for a very small cranial opening, 
leading inevitably to cortical herniation. It should be noted that surgical intervention for intracranial tumors was still held in disregard during that early period. As related by Cushing $^{8}$ almost a decade would pass before surgery began to increase in popularity, primarily thanks to various stimulating reports, such as that of von Monakow in 1900, in which he encouraged surgeons of that time to be less reluctant to perform cranial surgery.

Cohen $^{6}$ and Creed and Seeger ${ }^{7}$ state that in the early 1900s several surgical techniques were developed, some of which could clearly be considered ancestors to the modern procedures. In 1903, Woolsey performed a unilateral suboccipital craniectomy in one stage; 1 year later, as Cohen reports, Fraenkel would describe that technique in great detail. It was, however, Krause who became most closely associated with the suboccipital procedure, after performing a successful unilateral osteoplastic operation in two stages in $1905 .{ }^{7,9}$ The suboccipital approach has remained, after a great number of modifications, the standard route for neurosurgical access to the posterior fossa and the cerebellopontine angle, and is currently known as the retrosigmoid approach.

The high mortality rate associated with the unilateral suboccipital operation soon led to the search for other ways to access acoustic tumors. Cushing ${ }^{8}$ wrote that in 1904, Panse described a new technique entailing direct access to the tumor through the pyramidal bone, but this translabyrinthine approach never achieved widespread application due to the inevitable destruction of the middle ear structures and resulting loss of hearing, as well as its limited operating field, which forbade the excision of larger tumors. In an attempt to overcome the shortcomings of the previous procedures, several surgeons suggested various combined suboccipital and petrosal approaches, but none of these demonstrated clear benefits compared with the initially described one.

It was not until Harvey Cushing's era that surgical techniques for the exploration of the posterior fossa became standardized and extirpation of acoustic tumors was associated with an acceptable level of complications and mortality. Cushing ${ }^{8}$ stated in his clinical series in 1917 that the translabyrinthine approach had serious disadvantages and he advocated that the operation should not be considered the treatment of choice. The depth of the wound and the narrow field of action surrounded by important vascular structures, such as the sigmoid and superior petrosal sinuses as well as the carotid artery, limited the applicability of this procedure to very small intracanalicular tumors. Furthermore, Cushing believed that the translabyrinthine approach lacked any purpose as a palliative measure because removal of the tumor was always partial. Another drawback of that procedure was the high risk for meningitis to develop due to cerebrospinal fluid leakage through the wound or the ear. Cushing therefore concluded that the translabyrinthine technique was not optimal, acknowledging, however, the possibility of using that procedure for small intracanalicular tumors in the future if a more exact and early diagnosis could be obtained. Similarly, Cushing described the morbidity and mortality rates associated with the unilateral suboccipital approach as shocking, considering its future application only after effective measures against blood loss, injury, and increased intracerebral tension were developed. He proposed, therefore, a bilateral suboccipital approach aiming at subtotal intracapsular removal of acoustic neurinomas.

Although similar approaches had been attempted in the past, Cushing was the first to emphasize the importance of avoiding cortical herniation and medullary compression by making a large curvilinear incision extending between the two mastoid processes, approximately $4 \mathrm{~cm}$ above the occipital protuberance, followed by a midcervical incision continued to the spinous process of the upper cervical vertebrae. Despite its magnitude, this operation addressed effectively the issue of avoiding compressive effects on the brain, leading to a marked reduction in the complication and mortality rates. Furthermore, it allowed the exploration of the side contralateral to the suspected one, which frequently was proved to be occupied by the lesion; plain X-ray films, although sometimes diagnostic of the affected side by showing changes of the porus acusticus internus (as first suggested by Henschen in 1912), were far from being considered reliable. Cushing ${ }^{8}$ pointed out the importance of the correct placement of the patient in the face-down position so as to facilitate access to the involved surgical area. Controlling bleeding by applying pressure with the fingers and placement of clamps was emphasized. To reduce tension further, cerebrospinal fluid was secured from the posterior cistern, and, if that failed, puncture of the lateral ventricle could be used. Nevertheless, subtotal enucleation of the tumor was never curative and any attempt to remove a larger part of the mass would result in paralysis of the facial nerve.

In the years that followed, the introduction of newer diagnostic modalities and the advances in intraoperative technology would have tremendous impact on the neurosurgical care of patients. The electrosurgical unit and suction significantly ameliorated the surgical work, providing an effective control of intraoperative hemorrhage and keeping the operative field clear. ${ }^{22}$ Dandy invented ventriculography in 1918, and, 1 year later, pneumoencephalography. Angiography was discovered a few years later (1926) by Moniz and Lima, revolutionizing the diagnostic armamentarium of the neurosurgeon. According to Yaşargil, ${ }^{26}$ under these favorable new conditions, the first case of a total removal of an acoustic neurinoma with preservation of the facial nerve was reported by Cairns in 1931. The suboccipital approach was modified by Dandy, who advocated a small lateral suboccipital flap on the side of the lesion and total removal of the tumor, in contrast to the bilateral approach and subtotal resection suggested by Cushing. ${ }^{8}$ As he later recalled, Dandy's ${ }^{9}$ first successful total removal of an acoustic neurinoma was presented in 1917, the very same year that Cushing had published his series advocating a partial enucleation of the tumors and expressing his grave doubts about the feasibility of a safe total removal of these lesions. ${ }^{10}$ This controversy was later discussed by Dirks ${ }^{10}$ in a book chapter on acoustic neuromas. It is alleged that Dandy's reporting of tumor extirpation, without consulting Cushing first, severely traumatized their friendship.

As Yaşargil ${ }^{26}$ relates, Dandy's technique was further refined by Kenneth McKenzie and Eben Alexander ${ }^{18}$ and by Herbert Olivecrona. ${ }^{23}$ As stated by McKenzie and Alexander, the patient was now most often placed in the seated and not in the prone position, following the observations of Gardner in 1939 about the advantages of the former. The seated position would become the position of choice for 
many neurosurgeons, to be replaced in recent years by the three-quarters prone or the mostly supine position with the head turned to the opposite side.,.$^{2,6,25}$ Exposure and removal of part of the occipital bone was followed by elevation or resection, when indicated, of a segment of the cerebellum. ${ }^{6,23}$ Emphasis was laid on the careful dissection of the facial nerve to achieve, if possible, the preservation of its function; any arteries entering the tumor were to be clipped and divided to achieve better control of hemorrhage. This technique has been modified, ultimately entailing minimal removal of bone behind the sigmoid sinus combined with exposure of the intracanalicular portion of the tumor by removal of the posterior wall of the internal auditory canal (retrosigmoid transmeatal approach).

Overshadowing these other advances, the greatest breakthrough that marked the transition to modern neurosurgery was, without doubt, the introduction of the surgical microscope. It was first applied to acoustic neurinomas by an otological surgeon, William House, in $1961 .{ }^{14}$ As Yaşargil ${ }^{26}$ also attests, the operating microscope enabled the visualization of small structures such as nerves and vessels, contributing significantly to a more radical tumor resection and preservation of the facial nerve and inner ear structures, depending of course on the exact size and localization of the lesion. With the aid of the microscope, House introduced the translabyrinthine and middle fossa approaches for the removal of acoustic tumors, ${ }^{4,5,13-15,17}$ initial descriptions of which were reported as early as 1904. ${ }^{8,15}$ The translabyrinthine approach, comprising a postauricular incision, complete mastoidectomy, and labyrinthectomy, provided a wide tumor exposure with facial nerve preservation and is currently indicated for patients with large tumors in whom hearing loss is inevitable., ${ }^{2,3}$ The middle cranial fossa approach entails a craniotomy in the squamous part of the temporal bone and exposure of the geniculate ganglion, with bone removal up to the superior part of the internal auditory canal. ${ }^{2,15}$ It has become the procedure of choice for patients with small tumors extending no more than $5 \mathrm{~mm}$ into the cerebellopontine angle, in which cases hearing preservation is desirable. ${ }^{2,15}$ As reported in Kondziolka, et al., ${ }^{16}$ and Noren, ${ }^{20}$ a few years after the introduction of the surgical microscope, in 1969, Lars Leksell and Bjorn Meyerson performed the first gamma knife radiosurgical operation for acoustic neurinoma, a procedure that now constitutes a valid option for the treatment of these tumors.

In addition to the microscope, the development of new diagnostic modalities marked the transition to the modern era in the management of acoustic tumors. Positive contrast cisternography was introduced in the 1960s, allowing the detection of moderate and large tumors; as Creed and Seeg$\mathrm{er}^{7}$ relate, House and Hitselberger further refined the diagnosis of acoustic neurinoma with the introduction of the polytome Pantopaque technique in 1968 for the identification of small tumors in the internal auditory canal. Inability to fill the entire canal with Pantopaque raised the suspicion of an acoustic tumor. A few years later, the introduction of computerized tomography and especially that of magnetic resonance imaging revolutionized the detection of tumors of the cerebellopontine angle. An accurate early diagnosis could now be obtained, providing detailed information on the exact location and size of the tumor, thus facilitating prompt surgical intervention.

The advances in diagnostic technology and surgical tech- niques are clearly depicted in the prognosis and surgical outcome of patients with acoustic neuromas. The surgical mortality rate in the beginning of the 20th century ranged between 68 and $86 \%$, to be reduced approximately $20 \%$ only by the meticulous surgical technique of Harvey Cushing and the great emphasis he laid on the avoidance of herniation and the decrease of intracerebral tension during operation. ${ }^{8}$ Intraoperative death rates were further reduced to 10 to $20 \%{ }^{21,25}$ after the introduction of the electrosurgical unit and suction as well as the refinement of surgical techniques by Dandy, McKenzie, Olivecrona, and others. A significant contribution was made by the more precise localization of tumors, facilitated by ventriculography, pneumoencephalography, and angiography. In the 1960s, House managed to lower the intraoperative mortality rate to less than $10 \%,{ }^{25}$ with the introduction of the surgical microscope. Early detection of small tumors and accurate localization of the lesions and their anatomical borders, which was made possible by the computerized tomography and magnetic resonance imaging modalities as well as the perfection of microsurgical techniques, further reduced the intraoperative death rates to 0.8 to $5 \% .^{25}$

Preservation of the facial nerve is another aspect of acoustic neuroma surgery that benefited greatly from the advancements in diagnosis and surgical technique. According to Yaşargil, ${ }^{26}$ almost 20 years after the first case of facial nerve preservation was reported by Cairns in 1931, Givré and Olivecrona reported that this outcome was accomplished in $30 \%$ of their patients. The introduction of the surgical microscope, which enabled the intraoperative identification of small anatomical structures, and the development of the translabyrinthine approach by House in the 1960s were undoubtedly the turning points for successful facial nerve dissection and preservation. The detection of very small tumors on computerized tomography scans and, mainly, on magnetic resonance images led to further decreases in facial nerve disruption during tumor removal. Anatomical preservation of the facial nerve can now be achieved, depending on the size of the tumor, in more than $98 \%$ of patients, the majority of whom also attain preservation of facial nerve function., ${ }^{2,3}$

\section{CONCLUSIONS}

The development of acoustic neurinoma surgery, from the early attempts at the end of the 19th century until today's safe and efficacious surgical procedures, has been marked by the meticulous efforts of many dedicated surgeons. As with most advances in medicine, progress was gradual and stepwise and demanded intimate communi cation between the members of the surgical community throughout the world. The contribution of technology has been of paramount importance, radically altering the diagnosis and management of acoustic neurinomas.

\section{References}

1. Ballance CA: Some Points in the Surgery of the Brain and Its Membranes. London: Macmillan, 1907, p 276

2. Brackmann DE: Acoustic neuroma: surgical approaches and complications. Ann Acad Med Singapore 20:674-679, 1991

3. Briggs RJS, Fabinyi G, Kaye AH: Current management of acoustic neuromas: review of surgical approaches and outcomes. J Clin Neurosci 7:521-526, 2000 
4. Browne JD, Fisch U: Transotic approach to the cerebellopontine angle. Otolaryngol Clin North Am 25:331-346, 1992

5. Camins MB, Oppenheim JS: Anatomy and surgical techniques in the suboccipital transmeatel approach to acoustic neuromas. Clin Neurosurg 38:567-588, 1992

6. Cohen NL: Retrosigmoid approach for acoustic tumor removal. Otolaryngol Clin North Am 25:295-310, 1992

7. Creed L, Seeger JF: Radiologic evaluation of the acoustic neuroma. Ariz Med 41:739-743, 1984

8. Cushing H: Tumors of the Nervus Acusticus and the Syndrome of the Cerebellopontine Angle. Philadelphia: Saunders, 1917

9. Dandy WE: Results of removal of acoustic tumors by the unilateral approach. AMA Arch Surg 42:1026-1033, 1941

10. Dirks DD: Acoustic neuromas, in Youmans JR (ed): Neurological Surgery. Philadelphia: WB Saunders, 1982, Vol 5, pp 2967-3003

11. Eldridge R, Parry D: Vestibular schwannoma (acoustic neuroma). Consensus development conference. Neurosurgery 30: 962-964, 1992

12. Grossman RG, Loftus CM (eds): Principles of Neurosurgery, ed 2. Philadelphia: Lippincott-Raven, 1999

13. Hitselberger WE, House WF: Surgical approaches to acoustic tumors. Arch Otolaryngol 84:286-291, 1966

14. House WF: Surgical exposure of the internal auditory canal and its contents through the middle, cranial fossa. Laryngoscope 71:1363-1385, 1961

15. House WF, Shelton C: Middle fossa approach for acoustic tumor removal. Otolaryngol Clin North Am 25:347-359, 1992

16. Kondziolka D, Lunsford LD, Flickinger JC: Acoustic neuroma radiosurgery. Origins, contemporary use and future expectations. Neurochirurgie 50:427-435, 2004
17. Lambert PR: House: "surgical exposure of the of the internal auditory canal and its contents through the middle cranial fossa." (Laryngoscope 71:1363-1385, 1961). Laryngoscope 106:1195-1198, 1996

18. McKenzie KG, Alexander E Jr: Acoustic neuroma. Clin Neurosurg 2:21-36, 1954

19. National Institutes of Health Consensus Development Program: Acoustic neuroma. NIH Consensus Statements. (http://con sensus.nih.gov/cons/087/087_statement.htm) [Accessed 23 March 2005]

20. Noren G: Gamma knife radiosurgery of acoustic neurinomas. A historic perspective. Neurochirurgie 50:253-256, 2004

21. Olivecrona $\mathrm{H}$ : Analysis of results of complete and partial removal of acoustic neuromas. J Neurochem 13:271-272, 1950

22. Olivecrona H: Neurosurgery, past and present. Acta Neurochir 2:4-8, 1951

23. Olivecrona H: The removal of acoustic neurinomas. J Neurosurg 26:100-103, 1967

24. Stone JL: Sir Charles Ballance: pioneer British neurological surgeon. Neurosurgery 44:610-632, 1999

25. Wiet RJ, Teixido M, Liang JG: Complications in acoustic neuroma surgery. Otolaryngol Clin North Am 25:389-412, 1992

26. Yaşargil MG, Fox JL: The microsurgical approach to acoustic neurinomas. Surg Neurol 2:393-398, 1974

Manuscript received February 9, 2005.

Accepted in final form March 23, 2005.

Address reprint requests to: Kostas N. Fountas, M.D., Ph.D., Department of Neurosurgery, The Medical Center of Central Georgia, Mercer University, 840 Pine Street Suite 880, Macon, Georgia 31201. email: knfountasmd@excite.com. 